Proceedings of the Mew Zealand Grassland Association 51: 157-162 (1990)

\title{
An on-farm evaluation of white and subterranean clovers in North Island hill country
}

\author{
M.J. Macfarlane ${ }^{1,2}$ A. W. McGowan', G. W.Sheath', \\ and C.J. Korte ${ }^{3}$ \\ 1 Present address: Antarctic Division, DSIR, \\ PO. Box 13-247, Christchurch \\ ${ }^{2}$ MAF, Whatawhata Hill Country Research Centre, \\ Private Bag, Hamilton \\ ${ }^{3}$ MAF, Flock House, Private Bag, Bulls
}

ABSTRACT Five white clover and 5 subterranean clover cultivars were evaluated under 'on-farm' conditions at 21 North Island sites over 5 years. Seedlings were established in the field and their content in the sward followed by point analysis. Grasslands Huia, Grasslands Tahora and Whatawhata Early Flowering established most rapidly. Overall, white clover was not altered by the introduction of any cultivar into existing pastures. White clover content of the sward appear to be determined by factors other than genetic potential, such as other species present, current management practices, and climate. Al subterranean clover cultivars established populations, though Tallarook was the most successful. At most sites Tallarook regenerated the best, followed by Mt Barker. The contribution to legume density made by any other subterranean clover cultivar was minimal

K eywords white clover, subterranean clover, hill country, point analysis

\section{NTRODUCTION}

During 1981-1984 cultivars of white and subterranean clover were evaluated at 8 hill country sites throughout New Zealand (Chapman et al. 1986). These trials yielded preliminary information on the likely suitability of material in hill country. Because of the two techniques used, the experiments provided insufficient data to confidently recommend cultivars for the range of conditions found in New Zealand hill country.

White clover resident in hill country generally does not resemble Grasslands Huia (Macfarlane \& Sheath 1984), but is similar to the recently released Grasslands Tahora, a more suitable plant form. This cultivar and other white clover (Grasslands Pitau, Grasslands Kopu and Whatawhata Early Flowering) material had not been extensively evaluated under hill country conditions.

A similar situation existed for subterranean clover. Little or no data are available to justify general and/or specific recommendations other than the standard cultivars, Mt Barker and Tallarook.
The objective was to evaluate white and subterranean clover, either currently available or pending release, under 'on-farm' hill country conditions. A new technique was developed, based on point analysis, which aimed at assessing the additional contribution of introduced white and subterranean clover in the presence of a background population of either species.

\section{METHODS}

\section{$M$ aterial evaluated}

White clover material evaluated included the 4 available New Zealand cultivars plus material pending release at the beginning of the trial (Table 2). Subterranean clover cultivars evaluated were those in current or recent use, Tallarook, Mt Barker and Woogenellup, plus 2 other promising Australian cultivars, Nangella and Seaton Park.

White clover and subterranean clover comparisons were conducted independently. Where the legume was clearly inappropriate for that location, it was not assessed, e.g. sub clover on a summer moist site.

\section{Sites}

Twenty-one sites (Northland 2, Waikato 2, Rotorua/Bay of Plenty 3, Gisbome-East Coast 4, Hastings-East Coast 5, Taranaki 2, Central Plateau 3) were selected to be representative of the farming conditions of the area (slope, soil type, fertiliser, stock and management practices). Trial sites were unfenced and received no preferential treatment, i.e. 'on-farm' conditions. These sites covered a wide range of climatic and physical conditions (Table I), from swards that were white clover dominant (Marotiri and Te Kuiti), to mixtures of each (most sites), through to dominant subterranean clover (Wairoa) . During the trial, climate was similar to long term normals, except that during March 1986 monthly rainfall was only half the average, while for March 1987 rainfall was $80-100 \%$ higher than average (Table I).

\section{Technique}

The trials were establishing during the autumn of 1984 and 1985. Seedlings were raised in sterilised potting medium and inoculated with an appropriate Rhizobium strain. At each site, 24 individual $10 \mathrm{~m}$ lines were located (4 blocks x 5 cultivars plus one control line per block) for each species. The spacing between lines varied (1-2 $\mathbf{m})$. To reduce competition on establishing seedlings, lines were mown and/or hard grazed and sprayed with a growth retardant (maleic hydrazide 17 
product/ha) 1-4 weeks before planting (except all those on the East Coast which were not treated with growth retardant). Along each line, 12-week-old seedlings of one cultivar were transplanted at $20-\mathrm{cm}$ intervals. Control lines were left unplanted and received all preplanting treatments. Seedlings which failed to establish after 8 weeks were replaced.

All lines were assessed for clover density by point analysis (Radcliffe \& Mountier 1964), during September/October for 4-5 years after planting; no attempt was made to measure clover yield. Because of the nature of the point analysis technique, the density of larger-leaved clovers (e.g. Pitau and Kopu) may be overestimated slightly. Data for the first, third and last (normally fifth) years are presented (Tables 2 and 3). 200 points were measured down each line, and data are expressed as percentage total hits i.e. (total hits on clover through the swardlnumber of points) $\mathrm{x} 100$, for each species. Two to 3 weeks before pointing, sites were grazed or mowed where necessary to give a pasture height of $2-5 \mathrm{~cm}$ at pointing. Point analysis was done in early spring when pasture density is high and pasture length is short. The amount of clover in control lines is assumed to be that present in an unplanted control. Data were analysed to test for differences between cultivars (between cultivars, Tables 2 and 3 ) and for differences from the background population of either white or subterranean clover (control vs cultivars, Tables 2 and 3 ). Untransformed means are presented together with significances obtained from the most appropriate analysis.

\section{RESULTS AND DISCUSSION}

\section{White clover}

Huia, Tahora and Whatawhata Early Flowering established the most rapidly during the first year (Table
2). Between-cultivar or cultivar vs control differences were few. After the first year seldom were the levels of white clover in planted lines higher than in the unplanted controls. During the first years large-leaved cultivars could be distinguished from resident clovers in the sward. The gradual disappearance of largeleaved types (Kopu and Pitau) was soon compensated for by the occupancy of this space by resident clover. Korte \& Quilter (1990) reported rapid loss of feather mark Huia plants at 4 sites.

The amount of white clover in the swards appeared to reflect a 'natural' balance due to the prevailing management and climate. This indicates that white clover contribution to the sward was limited by factors such as other species present, current management practices and climate. White clover is a colonising species capable of rapidly tilling available spaces in the sward. Plant competition (especially at moist sites) and soil fertility would seem to have been able to control available space.

No cultivar was assessed as being more tolerant of summer drought. Possibly deeper-rooting plants (Kopu) were grazed out and competition reduced reestablishment from seed even for the strongly reseeding cultivar Whatawhata Early Flowering. Unfortunately, the measurement technique used reflected the annual balance of numerous stresses and was more closely linked to vegetative spread and persistence. The adaptability and durability of resident white clover populations were illustrated where clover populations in control lines at extremely summer-dry sites were able to re-establish themselves to previous levels after near total collapse after droughts (Ngatapa, Wairakei)

\section{Subterranean clover}

All subterranean clovers established well in the first year, Tallarook being the most vigorous (Table 3). At

Table 1 Site climate and physical data $\left({ }^{\prime}=\right.$ estimated, Slope $\left.\mathrm{E}=0-10^{\circ}, \mathrm{M}=10-20^{\circ}, \mathrm{S}=20^{\circ}\right)$.

\begin{tabular}{|c|c|c|c|c|c|c|c|c|c|c|c|}
\hline Site & $\mathrm{pH}$ & Olsen & $\mathbf{P}$ & $\mathbf{K}$ & Slope & Aspect & $\begin{array}{c}\text { Annual } \\
\text { rainfall } \\
\mathbf{m m}\end{array}$ & $\begin{array}{c}\text { Dec-Mar } \\
\text { rainfall } \\
\text { mm }\end{array}$ & $\begin{array}{c}\text { Deviatio } \\
\text { normal } \\
1985\end{array}$ & $\begin{array}{l}\text { from } \\
\text { or Dec-1 } \\
1986\end{array}$ & $\begin{array}{c}\text { rainfall } \\
\text { Mar }(\mathrm{mm}) \\
1987\end{array}$ \\
\hline Northland & 6.4 & 25 & & 8 & $M$ & $\mathrm{~N}$ & 1495 & 403 & $=$ & 218 & .279 \\
\hline Tinopai & 5.9 & 27 & & 15 & $\mathbf{M}$ & NW & 1495 & 403 & & 218 & .279 \\
\hline Whatrwhata A & 5.1 & 24 & & 1 & $s$ & $\mathrm{~N}$ & 1627 & 435 & -17 & 25 & 31 \\
\hline Whatawhata B & 5.2 & 15 & & 7 & $\mathbf{M}$ & $\mathrm{NW}$ & 1627 & 435 & -17 & 25 & 31 \\
\hline Te Kuiti A & 5.4 & 10 & & 5 & $\mathbf{M}$ & N W & 1547 & 447 & -10 & 54 & 58 \\
\hline $\begin{array}{l}\text { Ie Kninu A } \\
\text { Te Kuiti B }\end{array}$ & $\begin{array}{l}5.4 \\
5.6\end{array}$ & 9 & & 8 & $\mathrm{~S}$ & N W & 1547 & $\begin{array}{l}441 \\
447\end{array}$ & -10 & 54 & 58 \\
\hline Rotorua A & 5.7 & 10 & & 4 & $\vec{M}$ & $\mathrm{NE}$ & 1654 & 446 & 4 & .41 & 199 \\
\hline Rotonua B & 5.8 & 28 & & ; & $\mathbf{M}$ & MW & 1654 & 446 & 4 & -41 & 199 \\
\hline Rotonu C & 5.4 & 18 & & 5 & $\mathrm{E}$ & N W & 1780 & 569 & .60 & 35 & 91 \\
\hline Marotiri A & 5.1 & 90 & & 7 & $\mathrm{E}$ & $\mathrm{N}$ & $2000^{1}$ & 569 & -60 & 35 & 91 \\
\hline Mamtiri B & 5.2 & 50 & & 12 & $\mathbf{S}$ & $\mathrm{NE}$ & 2000 & 569 & -60 & 35 & 91 \\
\hline Wairakei & 5.2 & 30 & & 8 & $\mathbf{s}$ & $\mathrm{NE}$ & 1233 & 382 & 5 & -55 & 66 \\
\hline Tokomaru Bay & 5.3 & 19 & & 10 & s & S W & 1830 & 501 & 132 & .206 & .70 \\
\hline Matawai & 5.6 & 21 & & 6 & $E$ & $\mathrm{E}$ & 1925 & 686 & 70 & .298 & 106 \\
\hline Ngatapa & 5.6 & 7 & & 10 & $\mathbf{S}$ & N W & 110 & 333 & 77 & -148 & .6 \\
\hline Whiroa & 5.6 & 7 & & 4 & $\vec{E}$ & S W & 1489 & 474 & 55 & .201 & 24 \\
\hline Makahu & 5.8 & 13 & & 6 & $\mathbf{S}$ & $S \mathrm{~W}$ & $\begin{array}{l}1409 \\
1925\end{array}$ & 537 & .36 & 24 & 230 , \\
\hline Manaming & 5.5 & 10 & & 5 & $\vec{s}$ & N W & 1035 & & & & \\
\hline Porangahau A & 6.3 & 14 & & 17 & $\mathrm{E}$ & $\mathrm{NE}$ & 1008 & 303 & 23 & .51 & 56 \\
\hline Poranoapau B & 5.8 & 21 & & 16 & E & N E & 1008 & 303 & 23 & .51 & 56 \\
\hline Porangahau C & 5.4 & 24 & & 8 & $\mathrm{E}$ & $\mathrm{N} \mathrm{E}$ & 1008 & 303 & 23 & .51 & 56 \\
\hline Porangahau D & 6.2 & 13 & & 22 & $\mathbf{E}$ & N E & $\begin{array}{l}1000 \\
1008\end{array}$ & $\begin{array}{l}509 \\
303\end{array}$ & 23 & .51 & 56 \\
\hline
\end{tabular}




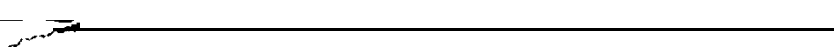

Table 2 White clover density after transplanting (Total hits per 100 points) Significance levels $*<0.05, * *<0.01$.

\begin{tabular}{|c|c|c|c|c|c|c|c|c|c|c|}
\hline Year & Year & Huia & Tahora & What EF & Pitau & Kopu & Control & SED & $\begin{array}{l}\text { Control } \\
\mathbf{v} \text { cultivars }\end{array}$ & $\begin{array}{l}\text { Between } \\
\text { cultivars }\end{array}$ \\
\hline Northland & $\begin{array}{l}84 \\
86 \\
87\end{array}$ & $\begin{array}{l}75.0 \\
40.5 \\
13.5\end{array}$ & $\begin{array}{l}76.3 \\
21.5 \\
15.3\end{array}$ & $\begin{array}{l}74.3 \\
23.2 \\
17.5\end{array}$ & $\begin{array}{c}55.8 \\
24.7 \\
10.8\end{array}$ & $\begin{array}{c}66.0 \\
\mathbf{1 4 . 5} \\
\mathbf{9 . 0}\end{array}$ & $\begin{array}{l}43.5 \\
35.0\end{array}$ & $\begin{array}{r}10.85 \\
7.04 \\
2.20\end{array}$ & & * \\
\hline Tinopai & $\begin{array}{c}84 \\
86 \\
88\end{array}$ & $\begin{array}{r}71.3 \\
13.5 \\
6.8\end{array}$ & $\begin{array}{r}71.5 \\
28.3 \\
8.8\end{array}$ & $\begin{array}{r}79.8 \\
28.8 \\
8.3\end{array}$ & $\begin{array}{r}61.7 \\
21.3 \\
7.3\end{array}$ & $\begin{array}{r}67.8 \\
27.3 \\
5.2\end{array}$ & $\begin{array}{r}21.5 \\
9.0\end{array}$ & $\begin{array}{r}14.70 \\
8.16 \\
1.90\end{array}$ & - & \\
\hline Whatrwhata A & $\begin{array}{l}85 \\
86 \\
88\end{array}$ & $\begin{array}{l}17.3 \\
20.8 \\
21.0\end{array}$ & $\begin{array}{l}19.6 \\
19.0 \\
22.1\end{array}$ & $\begin{array}{l}19.8 \\
20.4 \\
19.1\end{array}$ & $\begin{array}{l}16.8 \\
12.4 \\
20.1\end{array}$ & $\begin{array}{l}14.5 \\
13.2 \\
17.0\end{array}$ & $\begin{array}{l}12.2 \\
18.6 \\
21.0\end{array}$ & $\begin{array}{c}1.92 \\
6.95 \\
3.67\end{array}$ & $*$ & \\
\hline Whatawhata B & $\begin{array}{l}84 \\
86 \\
88\end{array}$ & $\begin{array}{c}25.9 \\
7.4 \\
11.5\end{array}$ & $\begin{array}{c}27.1 \\
13.4 \\
15.2\end{array}$ & $\begin{array}{l}34.3 \\
11.1 \\
14.1\end{array}$ & $\begin{array}{r}22.3 \\
6.6 \\
10.6\end{array}$ & $\begin{array}{l}22.2 \\
11.1 \\
13.6\end{array}$ & $\begin{array}{r}12.5 \\
1.5 \\
11.5\end{array}$ & $\begin{array}{l}2.72 \\
3.13 \\
2.50\end{array}$ & $\$$ & • \\
\hline Te Kuiti A & $\begin{array}{l}84 \\
86 \\
88\end{array}$ & $\begin{array}{r}30.8 \\
31.9 \\
6.3\end{array}$ & $\begin{array}{l}40.4 \\
25.8 \\
12.6\end{array}$ & $\begin{array}{r}28.2 \\
30.0 \\
6.1\end{array}$ & $\begin{array}{l}28.0 \\
35.0 \\
11.3\end{array}$ & $\begin{array}{r}19.4 \\
30.0 \\
8.7\end{array}$ & $\begin{array}{r}14.4 \\
28.0 \\
7.9\end{array}$ & $\begin{array}{l}4.28 \\
7.71 \\
2.23\end{array}$ & - $\otimes$ & $\bullet$ \\
\hline Te Kuiti B & $\begin{array}{l}84 \\
86 \\
88\end{array}$ & $\begin{array}{l}33.3 \\
32.0 \\
22.7\end{array}$ & $\begin{array}{l}33.6 \\
31.7 \\
21.6\end{array}$ & $\begin{array}{l}25.4 \\
34.2 \\
17.0\end{array}$ & $\begin{array}{l}27.6 \\
32.9 \\
20.9\end{array}$ & $\begin{array}{l}31.5 \\
23.0 \\
24.5\end{array}$ & $\begin{array}{l}10.0 \\
33.2 \\
22.4\end{array}$ & $\begin{array}{l}4.19 \\
4.26 \\
3.36\end{array}$ & - $\otimes$ & \\
\hline Rotorua A & $\begin{array}{l}84 \\
85 \\
87\end{array}$ & $\begin{array}{r}19.9 \\
39.8 \\
0.2\end{array}$ & $\begin{array}{r}30.3 \\
31.2 \\
0.9\end{array}$ & $\begin{array}{r}25.0 \\
38.5 \\
1.1\end{array}$ & $\begin{array}{r}24.9 \\
35.3 \\
1.0\end{array}$ & $\begin{array}{r}15.5 \\
36.9 \\
0.8\end{array}$ & $\begin{array}{r}3.4 \\
41.9 \\
0.8\end{array}$ & $\begin{array}{l}3.84 \\
4.16 \\
0.47\end{array}$ & & \\
\hline Rotorua B & $\begin{array}{l}84 \\
85 \\
87\end{array}$ & $\begin{array}{r}13.9 \\
8.9 \\
11.6\end{array}$ & $\begin{array}{l}18.3 \\
13.1 \\
11.5\end{array}$ & $\begin{array}{l}17.4 \\
14.6 \\
14.7\end{array}$ & $\begin{array}{l}15.6 \\
13.3 \\
12.2\end{array}$ & $\begin{array}{l}14.0 \\
11.3 \\
14.3\end{array}$ & $\begin{array}{c}2.2 \\
13.4 \\
11.8\end{array}$ & $\begin{array}{r}16.70 \\
2.92 \\
3.00\end{array}$ & & \\
\hline Rotorua C & $\begin{array}{l}84 \\
85 \\
87\end{array}$ & $\begin{array}{l}48.4 \\
44.8 \\
10.3\end{array}$ & $\begin{array}{l}61.8 \\
48.6 \\
15.3\end{array}$ & $\begin{array}{l}41.2 \\
36.2 \\
10.9\end{array}$ & $\begin{array}{l}49.4 \\
47.0 \\
12.3\end{array}$ & $\begin{array}{r}46.8 \\
44.7 \\
8.7\end{array}$ & $\begin{array}{r}24.5 \\
49.8 \\
8.5\end{array}$ & $\begin{array}{l}8.75 \\
4.30 \\
4.77\end{array}$ & & \\
\hline Marotiri & $\begin{array}{l}84 \\
86 \\
88\end{array}$ & $\begin{array}{c}20.5 \\
17.3 \\
20.1\end{array}$ & $\begin{array}{l}23.2 \\
15.8 \\
12.1\end{array}$ & $\begin{array}{l}23.0 \\
15.2 \\
22.3\end{array}$ & $\begin{array}{c}17.5 \\
24.9 \\
16.7\end{array}$ & $\begin{array}{l}19.2 \\
16.6 \\
26.7\end{array}$ & $\begin{array}{c}16.1 \\
13.8 \\
17.8\end{array}$ & $\begin{array}{l}3.46 \\
4.74 \\
4.84\end{array}$ & & \\
\hline Marotiri B & $\begin{array}{l}84 \\
86 \\
88\end{array}$ & $\begin{array}{c}20.5 \\
18.2 \\
14.0\end{array}$ & $\begin{array}{l}23.2 \\
27.4 \\
29.6\end{array}$ & $\begin{array}{l}23.0 \\
25.0 \\
29.1\end{array}$ & $\begin{array}{c}17.5 \\
22.3 \\
24.9\end{array}$ & $\begin{array}{l}19.7 \\
23.9 \\
25.5\end{array}$ & $\begin{array}{l}16.1 \\
17.5 \\
20.9\end{array}$ & $\begin{array}{l}3.33 \\
4.98 \\
5.20\end{array}$ & & \\
\hline Wairakei & $\begin{array}{l}84 \\
86 \\
88\end{array}$ & $\begin{array}{c}31.3 \\
15.2 \\
4.6\end{array}$ & $\begin{array}{r}35.3 \\
20.5 \\
4.1\end{array}$ & $\begin{array}{r}31.9 \\
25.1 \\
4.1\end{array}$ & $\begin{array}{c}29.9 \\
13.4 \\
3.9\end{array}$ & $\begin{array}{c}32.0 \\
18.8 \\
2.7\end{array}$ & $\begin{array}{r}20.8 \\
19.5 \\
6.4\end{array}$ & $\begin{array}{l}3.45 \\
5.41 \\
1.84\end{array}$ & 44 & \\
\hline Tokomaru Bay & $\begin{array}{l}85 \\
87 \\
88\end{array}$ & $\begin{array}{c}15.5 \\
2.1 \\
7.7\end{array}$ & $\begin{array}{r}18.5 \\
2.9 \\
7.8\end{array}$ & $\begin{array}{c}14.9 \\
1.8 \\
7.8\end{array}$ & $\begin{array}{c}18.7 \\
3.4 \\
8.1\end{array}$ & $\begin{array}{c}12.5 \\
3.3 \\
10.2\end{array}$ & $\begin{array}{l}5.4 \\
3.8 \\
9.5\end{array}$ & $\begin{array}{c}2.09 \\
1.30 \\
3.44\end{array}$ & & \\
\hline Matawai & $\begin{array}{l}85 \\
87 \\
88\end{array}$ & $\begin{array}{c}10.7 \\
21.0 \\
20.7\end{array}$ & $\begin{array}{c}11.8 \\
24.0 \\
20.0\end{array}$ & $\begin{array}{l}12.4 \\
24.7 \\
17.8\end{array}$ & $\begin{array}{l}11.5 \\
21.8 \\
19.0\end{array}$ & $\begin{array}{c}10.5 \\
24.6 \\
16.3\end{array}$ & $\begin{array}{c}4.8 \\
17.8 \\
19.0\end{array}$ & $\begin{array}{l}\mathbf{1 . 7 7} \\
1.71 \\
\mathbf{2 . 0 5}\end{array}$ & $\bullet$ & \\
\hline Ngatapa & $\begin{array}{l}85 \\
87 \\
88\end{array}$ & $\begin{array}{c}14.1 \\
3.8 \\
6.0\end{array}$ & $\begin{array}{r}11.9 \\
0.0 \\
7.0\end{array}$ & $\begin{array}{r}14.6 \\
2.7 \\
6.7\end{array}$ & $\begin{array}{r}13.8 \\
0.0 \\
8.2\end{array}$ & $\begin{array}{r}11.9 \\
0.0 \\
7.2\end{array}$ & $\begin{array}{l}5.5 \\
1.6 \\
4.8\end{array}$ & $\begin{array}{l}1.75 \\
2.42 \\
3.29\end{array}$ & $*$ & \\
\hline Wairoa & $\begin{array}{l}85 \\
87 \\
88\end{array}$ & $\begin{array}{r}13.4 \\
2.7 \\
11.9\end{array}$ & $\begin{array}{r}22.0 \\
3.8 \\
15.4\end{array}$ & $\begin{array}{r}12.9 \\
5.6 \\
14.0\end{array}$ & $\begin{array}{r}21.2 \\
6.9 \\
16.8\end{array}$ & $\begin{array}{c}15.1 \\
3.3 \\
15.9\end{array}$ & $\begin{array}{c}8.4 \\
6.3 \\
15.2\end{array}$ & $\begin{array}{l}2.60 \\
2.64 \\
3.27\end{array}$ & & \\
\hline Makahu & $\begin{array}{l}84 \\
86 \\
87\end{array}$ & $\begin{array}{l}10.0 \\
14.3 \\
26.0\end{array}$ & $\begin{array}{l}13.3 \\
13.0 \\
27.2\end{array}$ & $\begin{array}{l}16.2 \\
15.2 \\
30.2\end{array}$ & $\begin{array}{l}10.4 \\
14.7 \\
29.5\end{array}$ & $\begin{array}{l}10.6 \\
16.1 \\
27.1\end{array}$ & $\begin{array}{r}3.5 \\
18.0 \\
23.8\end{array}$ & $\begin{array}{l}3.12 \\
3.60 \\
3.74\end{array}$ & & \\
\hline Manaming & $\begin{array}{l}84 \\
86 \\
87\end{array}$ & $\begin{array}{c}16.4 \\
15.6 \\
25.1\end{array}$ & $\begin{array}{l}14.3 \\
19.3 \\
20.5\end{array}$ & $\begin{array}{l}16.0 \\
20.5 \\
23.6\end{array}$ & $\begin{array}{l}16.3 \\
21.8 \\
20.6\end{array}$ & $\begin{array}{l}14.1 \\
15.4 \\
13.7\end{array}$ & $\begin{array}{c}6.7 \\
13.1 \\
18.7\end{array}$ & $\begin{array}{l}3.51 \\
3.52 \\
4.19\end{array}$ & & \\
\hline Porangahau A & $\begin{array}{l}84 \\
86 \\
87\end{array}$ & $\begin{array}{r}10.8 \\
5.3 \\
1.0\end{array}$ & $\begin{array}{r}10.0 \\
12.7 \\
2.4\end{array}$ & $\begin{array}{r}10.8 \\
8.2 \\
2.3\end{array}$ & $\begin{array}{c}14.3 \\
11.4 \\
3.0\end{array}$ & $\begin{array}{r}15.0 \\
12.5 \\
1.6\end{array}$ & $\begin{array}{r}7.3 \\
10.3 \\
2.7\end{array}$ & $\begin{array}{l}2.77 \\
3.39 \\
1.26\end{array}$ & & \\
\hline Porangahau B & $\begin{array}{l}84 \\
86 \\
87\end{array}$ & $\begin{array}{c}11.3 \\
20.3 \\
10.7\end{array}$ & $\begin{array}{c}1.3 \\
20.8 \\
11.6\end{array}$ & $\begin{array}{r}13.3 \\
22.2 \\
8.7\end{array}$ & $\begin{array}{c}8.5 \\
18.3 \\
8.0\end{array}$ & $\begin{array}{c}14.0 \\
19.5 \\
9.9\end{array}$ & $\begin{array}{r}14.9 \\
16.0 \\
6.7\end{array}$ & $\begin{array}{l}2.62 \\
4.16 \\
1.79\end{array}$ & & \\
\hline Porangahau C & $\begin{array}{l}84 \\
85 \\
86 \\
87\end{array}$ & $\begin{array}{l}8.2 \\
3.4 \\
5.0 \\
2.0\end{array}$ & $\begin{array}{l}9.5 \\
1.7 \\
5.3 \\
1.3\end{array}$ & $\begin{array}{c}12.0 \\
0.9 \\
4.5 \\
0.3\end{array}$ & $\begin{array}{l}7.5 \\
1.4 \\
6.7 \\
3.9\end{array}$ & $\begin{array}{l}8.2 \\
1.4 \\
2.8 \\
2.6\end{array}$ & $\begin{array}{l}6.7 \\
2.3 \\
3.6 \\
2.7\end{array}$ & $\begin{array}{l}2.13 \\
1.20 \\
1.05 \\
0.80\end{array}$ & & \\
\hline Porangahau D & $\begin{array}{l}84 \\
86 \\
87\end{array}$ & $\begin{array}{r}11.5 \\
1.0 \\
0.7\end{array}$ & $\begin{array}{c}10.5 \\
1.3 \\
0.6\end{array}$ & $\begin{array}{l}7.3 \\
1.3 \\
1.3\end{array}$ & $\begin{array}{r}14.0 \\
1.1 \\
1.6\end{array}$ & $\begin{array}{r}14.3 \\
1.4 \\
0.9\end{array}$ & $\begin{array}{l}6.5 \\
1.1 \\
2.2\end{array}$ & $\begin{array}{c}3.42 \\
0.52 \\
1.24\end{array}$ & & \\
\hline
\end{tabular}


most sites all subterranean clover cultivars except Tallarook and to a lesser degree Mt Barker, failed to re-establish populations in successive seasons. At Wairoa, subterranean clover was already the predominant legume (Korte \& Quilter 1990), and the introduction of other cultivars made no significant contribution to clover density. At Rotorua and Te Kuiti white clover continued to be the most dominant legume and effectively excluded the establishment of further subterranean clover populations.
At the end of the trial and contributions made by Nangella, Woogenellup, and Seaton Park were minimal, possibly because of inadequate growth form and/or flowering characteristics of these cultivars for hill country conditions (Macfarlane \& Sheath 1984; Chapman et al. 1986). This further indicates that Tallarook-type cultivars (late flowering, dense crowning) are likely to be best suited to New Zealand hill country conditions.

Table 3 Subterranean clover density after transplanting (Total hits per 100 points). Significance levels $*<0.05$, $* *<0.01$.

\begin{tabular}{|c|c|c|c|c|c|c|c|c|c|c|}
\hline Site & $Y$ e a $r$ & Mt Barker & Nangella & a Seaton 1 & Tallarook & $\begin{array}{l}\text { Woogenel- } \\
\text { lup }\end{array}$ & Control & SED & $\begin{array}{c}\text { Control } \\
\text { v cultivars }\end{array}$ & $\begin{array}{l}\text { Between } \\
\text { cultivars }\end{array}$ \\
\hline Year & Year & Huia & Tahora W & Whata EF & Pitau & Kopu & Control & SED & $\begin{array}{l}\text { Control } \\
\mathbf{v} \text { cultivars }\end{array}$ & $\begin{array}{l}\text { Between } \\
\text { cultivars }\end{array}$ \\
\hline Northland A & $\begin{array}{l}84 \\
86 \\
87\end{array}$ & $\begin{array}{r}48.8 \\
1.0 \\
2.8\end{array}$ & $\begin{array}{r}55.6 \\
1.0 \\
2.8\end{array}$ & $\begin{array}{r}48.3 \\
0.7 \\
3.5\end{array}$ & $\begin{array}{r}79.3 \\
26.8 \\
4.0\end{array}$ & $\begin{array}{r}57.0 \\
32.0 \\
4.0\end{array}$ & $\begin{array}{l}5.0 \\
2.5 \\
6.5\end{array}$ & $\begin{array}{r}13.57 \\
11.23 \\
1.60\end{array}$ & ** & • \\
\hline Tinopai & $\begin{array}{l}84 \\
86 \\
88\end{array}$ & $\begin{array}{l}\mathbf{6 9 . 5} \\
\mathbf{3 8 . 8} \\
14.1\end{array}$ & $\begin{array}{r}71.3 \\
35.0 \\
2.1\end{array}$ & $\begin{array}{r}43.2 \\
37.8 \\
3.7\end{array}$ & $\begin{array}{l}91.0 \\
43.3 \\
16.7\end{array}$ & $\begin{array}{r}49.5 \\
42.5 \\
5.6\end{array}$ & $\begin{array}{l}2.0 \\
0.0 \\
2.4\end{array}$ & $\begin{array}{r}14.93 \\
15.60 \\
5.32\end{array}$ & i* & $\dot{\bullet}$ \\
\hline Whatawhata & $\begin{array}{l}84 \\
86 \\
\mathbf{8 8}\end{array}$ & $\begin{array}{c}11.6 \\
6.1 \\
5.1\end{array}$ & $\begin{array}{l}9.4 \\
1.3 \\
1.4\end{array}$ & $\begin{array}{l}6.5 \\
1.3 \\
1.5\end{array}$ & $\begin{array}{r}26.6 \\
5.6 \\
13.4\end{array}$ & $\begin{array}{c}17.7 \\
5.2 \\
6.6\end{array}$ & $\begin{array}{l}3.4 \\
\mathbf{0 . 5} \\
1: \mathrm{s}\end{array}$ & $\begin{array}{l}3.57 \\
2.78 \\
3.11\end{array}$ & ? & $* *$ \\
\hline Whatawhata B & $\begin{array}{l}84 \\
86 \\
88\end{array}$ & $\begin{array}{l}22.3 \\
11.7 \\
18.1\end{array}$ & $\begin{array}{r}25.5 \\
6.7 \\
14.8\end{array}$ & $\begin{array}{l}18.2 \\
11.8 \\
27.5\end{array}$ & $\begin{array}{r}28.5 \\
8.8 \\
19.0\end{array}$ & $\begin{array}{r}19.2 \\
4.6 \\
12.9\end{array}$ & $\begin{array}{c}2.8 \\
7.0 \\
18.5\end{array}$ & $\begin{array}{l}2.97 \\
3.00 \\
6.65\end{array}$ & $* *$ & • \\
\hline Te Kuiti A & $\begin{array}{l}84 \\
86 \\
88\end{array}$ & $\begin{array}{r}23.4 \\
3.5 \\
3.7\end{array}$ & $\begin{array}{r}27.4 \\
3.9 \\
0.3\end{array}$ & $\begin{array}{r}16.7 \\
3.6 \\
1.6\end{array}$ & $\begin{array}{r}32.6 \\
7.0 \\
5.6\end{array}$ & $\begin{array}{r}26.0 \\
2.7 \\
1.6\end{array}$ & $\begin{array}{l}0.5 \\
1.3 \\
0.3\end{array}$ & $\begin{array}{l}3.96 \\
3.23 \\
2.20\end{array}$ & 1 & • \\
\hline Te Kuiti B & $\begin{array}{l}84 \\
86 \\
88\end{array}$ & $\begin{array}{r}24.4 \\
1.4 \\
0.5\end{array}$ & $\begin{array}{r}19.7 \\
2.2 \\
0.0\end{array}$ & $\begin{array}{r}15.6 \\
2.5 \\
0.0\end{array}$ & $\begin{array}{r}35.0 \\
1.5 \\
2.4\end{array}$ & $\begin{array}{c}24.7 \\
1.3 \\
0.5\end{array}$ & $\begin{array}{l}0.0 \\
0.0 \\
0.0\end{array}$ & $\begin{array}{l}4.82 \\
1.36 \\
0.87\end{array}$ & '. & 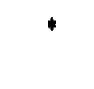 \\
\hline Rotorua A & $\begin{array}{l}84 \\
85 \\
87\end{array}$ & $\begin{array}{r}24.9 \\
4.2 \\
0.6\end{array}$ & $\begin{array}{r}30.8 \\
3.9 \\
2.5\end{array}$ & $\begin{array}{r}18.0 \\
0.7 \\
0.6\end{array}$ & $\begin{array}{l}38.2 \\
10.5 \\
0.8\end{array}$ & $\begin{array}{r}29.4 \\
3.8 \\
0.3\end{array}$ & $\begin{array}{l}0.0 \\
0.6 \\
0.0\end{array}$ & $\begin{array}{l}4.11 \\
1.77 \\
0.99\end{array}$ & 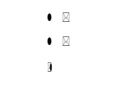 & * \\
\hline Wairakei & $\begin{array}{l}84 \\
86 \\
\mathbf{8 8}\end{array}$ & $\begin{array}{l}9.9 \\
0.4 \\
1.9\end{array}$ & $\begin{array}{r}12.1 \\
0.4 \\
0.4\end{array}$ & $\begin{array}{l}8.7 \\
1.1 \\
1.8\end{array}$ & $\begin{array}{r}20.4 \\
3.6 \\
6.7\end{array}$ & $\begin{array}{c}13.4 \\
0.9 \\
0.5\end{array}$ & $\begin{array}{l}0.6 \\
0.8 \\
1.5\end{array}$ & $\begin{array}{l}2.28 \\
1.32 \\
2.26\end{array}$ & • & 3 \\
\hline Tokomaru Bay & $\begin{array}{l}85 \\
86 \\
88\end{array}$ & $\begin{array}{c}18.1 \\
20.1 \\
12.7\end{array}$ & $\begin{array}{l}19.2 \\
21.9 \\
14.7\end{array}$ & $\begin{array}{l}16.8 \\
19.7 \\
14.5\end{array}$ & $\begin{array}{l}20.5 \\
21.7 \\
17.0\end{array}$ & $\begin{array}{l}15.2 \\
22.2 \\
12.5\end{array}$ & $\begin{array}{r}8.6 \\
14.8 \\
14.2\end{array}$ & $\begin{array}{l}2.36 \\
1.89 \\
4.13\end{array}$ & $*$ & \\
\hline Matzwai & $\begin{array}{l}86 \\
87 \\
88\end{array}$ & $\begin{array}{r}11.1 \\
7.6 \\
2.5\end{array}$ & $\begin{array}{r}11.6 \\
7.3 \\
6.2\end{array}$ & $\begin{array}{r}15.8 \\
1.5 \\
0.5\end{array}$ & $\begin{array}{r}17.1 \\
9.5 \\
6.2\end{array}$ & $\begin{array}{c}14.2 \\
6.1 \\
2.6\end{array}$ & $\begin{array}{l}1.7 \\
1.4 \\
1.3\end{array}$ & $\begin{array}{l}\mathbf{2 . 6 5} \\
\mathbf{2 . 0 0} \\
1.91\end{array}$ & $: \square$ & ' \\
\hline Ngatapa & $\begin{array}{l}85 \\
87 \\
88\end{array}$ & $\begin{array}{l}\mathbf{2 3 . 1} \\
\mathbf{1 3 . 8} \\
19.1\end{array}$ & $\begin{array}{l}22.7 \\
15.4 \\
17.8\end{array}$ & $\begin{array}{l}25.3 \\
14.6 \\
19.6\end{array}$ & $\begin{array}{l}27.3 \\
19.4 \\
26.0\end{array}$ & $\begin{array}{l}22.1 \\
12.7 \\
14.6\end{array}$ & $\begin{array}{l}13.7 \\
10.4 \\
20.3\end{array}$ & $\begin{array}{l}2.08 \\
2.35 \\
3.56\end{array}$ & $\stackrel{* *}{:}$ & : \\
\hline Wairoa & $\begin{array}{l}85 \\
87 \\
88\end{array}$ & $\begin{array}{r}31.3 \\
8.3 \\
25.2\end{array}$ & $\begin{array}{r}26.5 \\
8.3 \\
22.2\end{array}$ & $\begin{array}{r}33.3 \\
9.9 \\
22.7\end{array}$ & $\begin{array}{l}39.7 \\
10.2 \\
25.7\end{array}$ & $\begin{array}{r}32.5 \\
7.0 \\
20.8\end{array}$ & $\begin{array}{r}15.2 \\
8.8 \\
23.2\end{array}$ & $\begin{array}{l}5.42 \\
2.21 \\
4.31\end{array}$ & • & • \\
\hline Makahu & $\begin{array}{l}84 \\
85 \\
87\end{array}$ & $\begin{array}{r}18.6 \\
5.4 \\
2.1\end{array}$ & $\begin{array}{r}17.1 \\
2.2 \\
4.8\end{array}$ & $\begin{array}{l}4.3 \\
2.1 \\
0.3\end{array}$ & $\begin{array}{r}22.3 \\
5.9 \\
17.8\end{array}$ & $\begin{array}{r}10.7 \\
0.4 \\
4.1\end{array}$ & $\begin{array}{l}0.0 \\
0.0 \\
0.0\end{array}$ & $\begin{array}{l}2.06 \\
1.18 \\
3.36\end{array}$ & ** & 3 \\
\hline Porangahau A & $\begin{array}{l}84 \\
85 \\
88\end{array}$ & $\begin{array}{c}12.0 \\
18.6 \\
4.1\end{array}$ & $\begin{array}{r}12.0 \\
20.5 \\
3.1\end{array}$ & $\begin{array}{r}17.5 \\
17.9 \\
5.5\end{array}$ & $\begin{array}{r}13.8 \\
27.2 \\
8.8\end{array}$ & $\begin{array}{r}9.5 \\
12.1 \\
4.9\end{array}$ & $\begin{array}{l}6.3 \\
9.5 \\
1.8\end{array}$ & $\begin{array}{l}6.26 \\
6.44 \\
1.64\end{array}$ & * & * \\
\hline Porangahau B & $\begin{array}{l}85 \\
86 \\
88\end{array}$ & $\begin{array}{c}10.8 \\
12.9 \\
8.2\end{array}$ & $\begin{array}{l}8.2 \\
7.3 \\
5.7\end{array}$ & $\begin{array}{l}4.4 \\
6.4 \\
5.1\end{array}$ & $\begin{array}{c}16.5 \\
13.5 \\
9.1\end{array}$ & $\begin{array}{l}7.1 \\
9.9 \\
5.3\end{array}$ & $\begin{array}{l}7.9 \\
4.5 \\
6.0\end{array}$ & $\begin{array}{l}3.94 \\
3.10 \\
1.99\end{array}$ & * & • \\
\hline Porangahau C & $\begin{array}{l}84 \\
86 \\
\mathbf{8 8}\end{array}$ & $\begin{array}{r}15.5 \\
7.6 \\
4.3\end{array}$ & $\begin{array}{r}12.8 \\
10.5 \\
5.0\end{array}$ & $\begin{array}{r}11.8 \\
6.0 \\
3.6\end{array}$ & $\begin{array}{r}12.0 \\
13.9 \\
5.8\end{array}$ & $\begin{array}{r}15.5 \\
8.9 \\
4.6\end{array}$ & $\begin{array}{r}11.1 \\
\mathbf{6 . 1} \\
\mathbf{3 . 0}\end{array}$ & $\begin{array}{l}3.43 \\
2.86 \\
1.05\end{array}$ & . & \\
\hline Porangahau D & $\begin{array}{l}84 \\
86 \\
88\end{array}$ & $\begin{array}{c}11.3 \\
0.0 \\
0.3\end{array}$ & $\begin{array}{l}9.5 \\
0.2 \\
0.4\end{array}$ & $\begin{array}{c}9.3 \\
0.0 \\
0.8\end{array}$ & $\begin{array}{r}15.0 \\
0.2 \\
0.8\end{array}$ & $\begin{array}{l}8.5 \\
0.0 \\
1.4\end{array}$ & $\begin{array}{l}9.8 \\
0.0 \\
0.9\end{array}$ & $\begin{array}{l}2.99 \\
0.11 \\
0.54\end{array}$ & & \\
\hline
\end{tabular}




\section{Technique}

Application of the growth retardant, maelic hydrazide, appeared to suppress resident vegetation for up to 6 months. Between-year comparisons of white clover density within control lines would indicate that resident white clover was a suppressed species in the first year (Table 2). However, the impact of the retardant was short-lived as resident populations recovered to levels determined by the opportunity to occupy space at each site.

The establishment technique used in this experiment provided a good method for comparing the ability of cultivars to spread when competition is low. The first year's data reflect differences in the rate of establishment of a cultivar. The ability of the method to detect between-cultivar differences indicates its usefulness for identifying cultivar superiority in colonising an area.

If the addition of any planted material increased clover content above that of the resident background population, then it was assumed that the introduction of the cultivar was beneficial. The technique worked well for assessing the relative success of subterranean clover cultivars at sites where it was a suitable species. The approach was less successful for white clover because of the resilience of the resident white clover populations. Further, restricting density assessment to the original transplant line would have emphasised vegetative spread and persistence differences and underestimated the impact of white clover reseeding. Unlike subterranean clover that reseeds in close proximity to the parent plant there was no guarantee that under grazing white clover seed was shed or deposited within the measurement zone.

The technique has some limitations, but does provide a valuable and relatively easy method for the evaluation of clovers during establishment under realistic farm conditions, particularly where more traditional trial designs, are not possible, e.g. because of steepness or uniformity of land. It is particularly well suited to long term trials and 'on-f\&-m' trials where trial grazing management is impracticable.

\section{CONCLUSIONS}

The introduction of available new genetic material of white clover into resident white clover populations in hill country is unlikely to be of benefit. White clover content seems to be determined by site factors rather than genetic potential of currently available material.

Until new cultivars with Tallarook-type characteristics become available, Tallarook and Mt Barker are the only subterranean clover cultivars that can be recommended for introduction into North Island hill country.

Acknowledgements The authors would like to acknowledge the following for assistance in establishing and managing trials; G. Piggot, C. Smart, M. Fraser, B. Thorrold, G. Crouchley, G. Rys and A. Roberts and B. Dow for statistical assistance

\section{REFERENCES}

Chapman, D.F. ; Sheath, G. W. ; Macfarlane, M.J. ; Rumball, P.J.; Cooper, B.M.; Croucley, G.; Hoghmd, J.H.; Widdup, K.H. 1986. Performance of subterranean and white clover varieties in dry hill country. Proceedings of the N. Z. Grassland Association 47: 53-62

Korte, C.J.; Quilter, S.J. 1990. Effects of summer grazing and fertiliser on the clover content of hill country sward in the Gisborne region. Proceedings of the N.Z. G rassland Association 51: 00-00

Macfarlane M.J.; Sheath G.W. 1984. Clover - what types for dry hill country? Proceedings of the N.Z. G rassland Association 45: 140-150.

Radcliffe, J.E.; Mountier, N.S. 1964. Problems in measuring pasture composition in the field. Part 1. Discussion of general problems and some considerations of the point method. $N, Z$. journal of botany 2: $2: 90-97$. 